\title{
PERCEPCIÓN DE LA IMAGEN CORPORAL EN UNIVERSITARIAS DE LA CIUDAD DE BARRANQUILLA, COLOMBIA: UN ESTUDIO DESCRIPTIVO-TRANSVERSAL
}

Body Image Perception in University Students from Barranquilla, Colombia: A Descriptive-Cross-Sectional Study

Dolores Nieto-Ortiz, ${ }^{1}$ Isaac Nieto-Mendoza ${ }^{2}$

Correo electrónico: icnieto@mail.uniatlantico.edu.co

1. Magister en Educación. Facultad de Educación. Universidad del Atlántico, Barranquilla, Colombia. (Barranquilla, Colombia)

2. Filósofo. Universidad del Atlántico, Barranquilla, Colombia. (Barranquilla, Colombia)

Recibido: 05/04/2020 Aceptado: 20/05/2020

(c) (i) () 


\section{RESUMEN}

INTRODUCCIÓN. La preocupación por el peso y la imagen corporal surge tanto en hombres como en mujeres; no obstante, diversos estudios han demostrado mayor inquietud sobre la percepción corporal en mujeres. La preocupación extrema por la imagen corporal puede conllevar —en la mayoría de los casos - a trastornos alimentarios, acciones extremas para estar delgadas, miedo al rechazo y a las comparaciones, sintiéndose además imposibilitadas para ser amadas. OBJETIVo. Caracterizar la percepción de la imagen corporal de mujeres del programa Licenciatura en Educación Especial de la Universidad del Atlántico en el semestre 2019-2, Barranquilla, Colombia. METodoLOGía. Estudio descriptivo-transversal, llevado a cabo con 150 mujeres de la Universidad del Atlántico, aplicando el Body Shape Questionnaire (BSQ), con el fin de conocer su percepción de imagen corporal, teniendo en cuenta dos criterios: la insatisfacción corporal y la preocupación por el peso. RESULTADOS Y CONCLUSIONES. Se evidencia una tendencia de las mujeres hacia la preocupación extrema por la imagen corporal (64,7\%), lo que indica insatisfacción corporal y preocupación por el peso en las universitarias estudiadas. Esto representa un factor de riesgo para el padecimiento de trastornos emocionales como depresión, ansiedad y conducta asocial; no obstante, estos resultados representan un punto de partida para la creación de campañas de sensibilización y el fomento de estilos de vida saludables, disuadiendo acciones desesperadas que puedan atentar contra su salud física y mental.

Palabras clave: imagen corporal; insatisfacción corporal; preocupación por el peso; universitarias; estilos de vida saludables.

\section{ABSTRACT}

INTRODUCTION. Concern about weight and body image arises in both men and women. However, several studies have shown greater concern about body perception in women. Extreme preoccupation with body image can lead - in most cases - to eating disorders, extreme actions to be thin, fear of rejection and comparisons, also feeling unable to be loved. $\underline{\text { AIM. }}$. To characterize the perception of the body image of women from the Bachelor of Special Education program of the Universidad del Atlántico in the 2019-2 semester, Barranquilla, Colombia. METHODOLOGY. Descriptive-cross-sectional study, carried out with 150 women from the Universidad del Atlántico, applying the Body Shape Questionnaire (BSQ) in order to know their perception of body image, taking into account two criteria: body dissatisfaction and weight concern. $\underline{\text { RESULTS }}$ AND CONCLUSIONS. Women show a tendency towards extreme concern for body image (64.7\%), which indicates body dissatisfaction and weight concern in the university students that participated in the study. This represents a risk factor for emotional disorders such as depression, anxiety and asocial behavior. However, these results represent a starting point for the creation of awareness campaigns and the promotion of healthy lifestyles, discouraging desperate actions that could threaten their physical and mental health.

Keywords: Body Image; Body Dissatisfaction; Weight Concern; University; Healthy Lifestyles. 


\section{INTRODUCCIÓN}

La preocupación sobre el peso y la imagen corporal es preeminente en toda la población. De acuerdo con la American Psychiatric Association (1994), el exceso de preocupación por la imagen corporal trae consigo implicaciones en las conductas alimentarias de los individuos; esta insatisfacción con la imagen corporal, según Raich (2004), es más frecuente en mujeres, las cuales, afectadas por las burlas, la autopercepción negativa sentirse feas o deformes - trae consigo no solo repercusiones negativas a nivel alimentario, sino también «consecuencias sociales negativas como rechazos, comparaciones y, lo más importante, la imposibilidad de ser amadas» (Raich, 2004, p. 19).

Diversas investigaciones han demostrado que aquellas mujeres con creencias y pensamientos persistentes con respecto a su imagen corporal se han catalogados a sí mismas como obesas o con exceso de peso, que terminan siendo, más bien, ideas sobrevaloradas y absurdos que se alejan de la realidad (González-González de Mesa et al., 2016; Guzmán-Saldaña et al., 2017; Trejo-Ortiz et al., 2016; Zueck-Enríquez et al., 2016; Soto-Montero, Guevara-Valtier y Valdez-Montero, 2017; Sim-Sim et al., 2018). Asimismo, es preciso destacar la incidencia de los cánones actuales de belleza, que están sustentados en la delgadez como un criterio determinante con respecto a la imagen corporal asertiva (Guzmán-Saldaña et al., 2017).

La problemática que llama la atención en relación con una percepción de la imagen corporal negativa en mujeres según la OMS tiene que ver con los «trastornos alimentarios», que conllevan graves alteraciones en las conductas alimentarias y el índice de masa corporal, trayendo consigo además «consecuencias psicológicas, físicas y sociales adversas» (López y Treasure, 2012, p. 86). Es así como la «imagen corporal distorsionada» incide en la autoestima femenina, induciendo, según investigaciones, a la alimentación extremadamente restringida e intentos constantes por adelgazar y no querer mantener un peso normal o saludable, recurriendo a prácticas desesperadas con el fin de sentirse bien consigo mismas y que — muchas veces - conllevan problemas de salud corporal y mental (Duno y Acosta, 2019; Rincón-Barreto et al., 2019; Santos et al., 2017).

La problemática en América Latina, de acuerdo con Amezcua (2014), muestra que «9 de cada 10 personas que presentan desórdenes alimenticios son mujeres» (p. 3), donde México, Colombia, Venezuela y Argentina son líderes en indicadores relacionados con 
trastornos alimentarios asociados a la anorexia, siendo el común denominador entre las mujeres que sufren estos trastornos una baja autoestima y «el no aceptarse como son y nunca estar conformes con cómo se ven» (Amezcua, 2014, p. 4).

En Colombia, los estudios realizados a mujeres con respecto a su imagen corporal dan cuenta de una sobrestimación de su peso, asociadas con ingestas alimentarias basadas en alimentos de alto contenido grasoso, bebidas azucaradas y consumo de dulces y fritos. Asimismo, se evidencia que al realizar medidas antropométricas — peso y talla para Índice de Masa Corporal— se hace palmaria una tendencia a indicadores normales de masa corporal (Espinosa-Lara, 2014; Lara-Cervantes y Nieto-Mendoza, 2019; SalazarDuque, 2015; Florez-Alvarez, 2017).

En el departamento del Atlántico, de acuerdo con la investigación realizada por LaraCervantes y Nieto-Mendoza (2019), la sobrestimación de la imagen corporal en mujeres es preeminente; a pesar de reconocer las mujeres sus necesidades a nivel alimentario y actividad física en aras de sentirse bien consigo mismas, discrepan de adaptar a su estilo de vida conductas asertivas relacionadas con la alimentación y la práctica de actividad física moderada o vigorosa debido a que esta resulta ser una alternativa a «largo plazo», por lo cual, recurren a alternativas «de corto plazo» que implican - muchas veces«riesgos quirúrgicos debido al consumo de suplementos nutricionales que son usados como reemplazo de la alimentación diaria» (Lara-Cervantes y Nieto-Mendoza, 2019, pp. 114-115).

En la ciudad de Barranquilla, Colombia, la investigación realizada por Borda-Pérez et al. (2016) dio cuenta de una distorsión de la imagen corporal desde la edad adolescente, discrepando de indicadores de masa corporal que reflejan un estado normal. Debe tenerse en cuenta, de acuerdo con esta investigación, que en la adolescencia se presentan:

Profundos cambios físicos y emocionales en el ser humano, desarrollándose un gran interés por la imagen corporal, y se observa que la percepción de la misma puede estar distorsionada por un conjunto de factores de riesgo propios de la adaptación y decapitación de los adolescentes a su ambiente. (p. 473)

Por su parte, en mujeres barranquilleras, de acuerdo con una investigación realizada por la Universidad del Norte en instituciones educativas femeninas, se observó una tendencia del 44,3\% de adolescentes que tenían alto riesgo de desarrollar bulimia, reflejando además «alteraciones en el estado emocional tales como ansiedad en el 59,7\%, 
depresión en el 68,6\% e insatisfacción con la imagen corporal en el 92,1\%» (Borda-Pérez, 2016, p. 475).

La Universidad del Atlántico es uno de los entornos académicos que alberga un gran número de estudiantes en la Región Caribe (Universidad del Atlántico, 2017), por lo cual resulta un entorno de interés para el abordaje de este tipo de investigaciones, teniendo presente además lo que se hace palmario en este entorno con respecto a los problemas alimentarios identificados en la investigación de Nieto-Ortiz y Torrenegra-Ariza (2019) y Nieto-Ortiz y Nieto-Mendoza (2020), en la que se apreció un gran número de estudiantes con exceso de peso y obesidad, además de estilos de vida poco saludables. Cabe afirmar que, hasta el año 2019, no se han reportado estudios que busquen identificar la percepción de la imagen corporal en universitarias, lo que brinda mayor importancia a esta investigación.

Por tanto, la investigación se ha fundamentado en conocer la percepción de la imagen corporal del programa Licenciatura en Educación Especial, teniendo presente que, casi en su totalidad, la carrera tiene una preferencia por parte de las mujeres, además que está distanciada de carreras en las que se pone como supuesto que las estudiantes tengan conocimientos con respecto a los estilos de vida saludables y que son cursadas en su mayoría por mujeres como nutrición y dietética. Por tanto, este estudio pretende caracterizar la percepción de la imagen corporal de mujeres del programa Licenciatura en Educación Especial de la Universidad del Atlántico en el semestre 2019-2, Barranquilla, Colombia.

\section{MATERIAL Y MÉTODO}

Estudio descriptivo de corte transversal, realizado en universitarias de la Facultad de Educación de la Universidad del Atlántico, Barranquilla, Colombia, en el periodo académico 2019-2. Para la participación en el estudio se tuvieron en cuenta como criterios de inclusión que fuesen mujeres adscritas al programa Licenciatura en Educación Especial de la Universidad del Atlántico, matriculadas en el periodo 2019-2; por tanto, el estudio tuvo como muestra a 150 universitarias, seleccionadas de una población de 312 mujeres matriculadas en el semestre 2019-2 a través del procedimiento no probabilístico por conveniencia, representando un índice de participación del 48\%. 
Para la aplicación del cuestionario fue preciso contactar a las universitarias por medio de la búsqueda activa en espacios alternos a las jornadas académicas, a fin de no intervenir con su actividad educativa. Las participantes recibieron la información referente a los intereses de la investigación y sus alcances, para luego proceder a la firma del consentimiento informado y dar respuesta al cuestionario que integró el instrumento de este estudio.

El instrumento utilizado en la investigación fue el Body Shape Questionnaire (BSQ) — cuestionario de la figura corporal, en español—, haciendo uso de la validación realizada por Castrillón-Moreno et al. (2007) y utilizada en población colombiana, basada en el diseño original de Cooper et al. (1987), con un Alpha de Cronbach de 0.95, conformado por 34 ítems que se responden por medio del tipo «escala de Likert» de 6 puntos, cuyas opciones van de «nunca» hasta «siempre»; el puntaje mínimo es de 34 y el máximo de 204.

Para la presentación de los resultados, se clasificaron las preguntas teniendo en cuenta dos factores que fueron, «insatisfacción corporal» y «preocupación por el peso», tal y como se muestra en la Tabla 1.

Tabla 1. Descripción de factores

\begin{tabular}{|l|l|c|}
\hline Factores & \multicolumn{1}{|c|}{ Descripción } & \multicolumn{1}{c|}{ Número de pregunta } \\
\hline Factor 1 & Insatisfacción corporal & $1,3,7,8,10,11,12,13,14,15,17,18,19,20,25,26,27,29,31$ y 32 \\
\hline Factor 2 & Preocupación por el peso & $2,4,5,6,9,16,21,22,23,24,28,30,33$ y 34 \\
\hline
\end{tabular}

Fuente: Tomado de Castrillón-Moreno et al. (2007)

Para la valoración de la percepción de la imagen corporal general, se tuvieron en cuenta los baremos utilizados en la investigación de Lara-Cervantes y Nieto-Mendoza (2019) con población universitaria de la ciudad de Barranquilla, los cuales fueron: No preocupado — puntuación <81—, leve preocupación —-puntuación entre 81 y 110—, preocupación moderada —entre 111 y 140 - y preocupación extrema —puntuación $>140$ -

Tras la aplicación del instrumento, el análisis estadístico de los resultados se efectuó por medio del programa IBM SPSS V. 23 para Windows 10. La interpretación de los datos fue por medio del análisis univariado, midiendo las variables en escala nominal y describiendo los datos mediante frecuencia, mostrando la información en tablas, 
describiendo las variables numéricas a través de promedios, valor máximo y valor mínimo.

Por último, en cuanto a las consideraciones legales de la investigación, esta se acogió a los aspectos mencionados en la Resolución 8430 del Ministerio de Salud y Protección Social de Colombia (1993), especialmente en aquellos aspectos referentes a la solicitud de consentimiento informado, la protección de la identidad y la clasificación del estudio de acuerdo con sus implicaciones para con la integridad del individuo, catalogando la que corresponde a este estudio como sin riesgo.

\section{RESULTADOS}

\section{Características sociodemográficas}

Se estudiaron un total de 150 universitarias, con una mediana de edad de 19 años, siendo el rango de edad de mayor preeminencia 18-21 años (38,7\%) y con menor 22-25 años (30\%). El estrato socioeconómico de mayor frecuencia fue el «estrato 1» —bajo(59,3\%), es decir, mujeres de escasos recursos y el de menor el «estrato 4» —alto—, que alberga mujeres con mayores recursos económicos (3,3\%).

En cuanto al semestre académico, hubo mayor tendencia en el rango 7-8 (41,3\%) y menor en 1-3 (19,3\%).

Con respecto a la percepción de imagen corporal de las universitarias, como se muestra en la Tabla 2, se evidenció mayor tendencia en la «preocupación extrema de la imagen corporal»(64,7\%), seguido de «preocupación moderada» (23,3\%), «leve preocupación» (7,3\%) y, por último, «no preocupado» $(4,7 \%)$. 
Tabla 2. Aspectos sociodemográficos de la muestra estudiada

\begin{tabular}{|c|c|c|}
\hline Criterios & n & $\%$ \\
\hline \multicolumn{3}{|l|}{ Edad } \\
\hline $18-21$ & 58 & $38,7 \%$ \\
\hline $22-25$ & 45 & $30,0 \%$ \\
\hline $26-29$ & 47 & $31,3 \%$ \\
\hline \multicolumn{3}{|l|}{ Estrato } \\
\hline 1 (bajo) & 89 & $59,3 \%$ \\
\hline 2 (medio-bajo) & 43 & $28,7 \%$ \\
\hline 3 (medio) & 13 & $8,7 \%$ \\
\hline 4 (alto) & 5 & $3,3 \%$ \\
\hline \multicolumn{3}{|l|}{ Semestre } \\
\hline $1-3$ & 29 & $19,3 \%$ \\
\hline $4-6$ & 59 & $39,3 \%$ \\
\hline $7-8$ & 62 & $41,3 \%$ \\
\hline \multicolumn{3}{|l|}{ BSQ } \\
\hline No preocupado $<81$ & 7 & $4,7 \%$ \\
\hline Leve preocupación & 11 & $7,3 \%$ \\
\hline Preocupación moderada & 35 & $23,3 \%$ \\
\hline Preocupación extrema & 97 & $64,7 \%$ \\
\hline
\end{tabular}

Fuente: Elaboración propia

\section{Insatisfacción corporal de las universitarias}

En la Tabla 3, se muestran los resultados con respecto a la insatisfacción corporal, donde las universitarias indagadas aseveraron, en su mayoría, siempre sentirse molestas al hacerse exámenes de su aspecto físico (39,3\%), pensar que tienen glúteos, cadera, cintura o muslos demasiado grandes (84,7\%), se han sentido mal con su figura llorando por ello (73,3\%), evitado correr para que no les saltara la piel (50,7\%), sentirse gordas por el consumo de hasta una pequeña cantidad de comida (68\%), fijarse en la figura de otros y contrastarla con la suya de manera desfavorable para sí misma (86\%), ha interferido en la capacidad de concentración pensar en su figura (78\%), sentirse gordas mientras se duchan (96,7\%), evitan llevar ropa que marque su figura (75,3\%), sentirse gordas al comer dulces, pasteles u otros alimentos (92,7\%), ha evitado ir a actos sociales por sentirse mal con respecto a su figura (85,3\%), sentirse excesivamente gordas o llenas (79,3\%), acomplejadas por su cuerpo (92\%), pensado que es injusto que otras mujeres se vean más delgadas que ellas (96,7\%), preocuparse por ocupar demasiado espacio cuando están al lado de otras personas (84\%), sentirse mal con respecto a su figura al estar frente al espejo (92,7\%) y evitado situaciones en las cuales la gente pudiera ver su cuerpo (88\%). 
Por su parte, en cuanto a la preocupación por el hecho de que sus muslos se ensanchen cuando se sientan, el 37,3\% aseveró casi nunca preocuparse; asimismo, con respecto a si han vomitado para sentirse más delgadas y si han tomado laxantes para el mismo fin, las universitarias afirman «nunca» haber recurrido a estas acciones (80,7\% y 92,7\%, respectivamente).

Tabla 3. Criterios de insatisfacción corporal

\begin{tabular}{|c|c|c|c|c|c|c|}
\hline Ítem & Nunca & Es raro & A veces & $\begin{array}{c}\text { A } \\
\text { menudo }\end{array}$ & $\begin{array}{c}\text { Muy a } \\
\text { menudo }\end{array}$ & Siempre \\
\hline & $\mathrm{n}(\%)$ & n (\%) & $\mathrm{n}(\%)$ & n (\%) & $\mathrm{n}(\%)$ & $\mathrm{n}(\%)$ \\
\hline $\begin{array}{l}\text { 1. ¿Me siento molesta o enfadada al hacer un } \\
\text { examen de mi aspecto físico? }\end{array}$ & $27(18)$ & $\begin{array}{c}22 \\
(14,7)\end{array}$ & $15(10)$ & $5(3,3)$ & $0(0)$ & $59(39,3)$ \\
\hline $\begin{array}{l}\text { 3. ¿Pensé que mis nalgas, caderas, cintura o } \\
\text { muslos eran demasiado "grandes» gordos en } \\
\text { relación con el resto de mi cuerpo? }\end{array}$ & $0(0)$ & $0(0)$ & $3(2 \%)$ & $0(0 \%)$ & $\begin{array}{c}22 \\
(14,7)\end{array}$ & $127(84,7)$ \\
\hline $\begin{array}{l}\text { 7. ¿Te has sentido tan mal con tu figura que has } \\
\text { llorado por ello? }\end{array}$ & $5(3,3)$ & $0(0)$ & $20(13,3)$ & $0(0)$ & $15(10)$ & $110(73,3)$ \\
\hline 8. ¿Has evitado correr para que tu piel no saltara? & $\begin{array}{c}17 \\
(11,3)\end{array}$ & $12(8)$ & $5(3,3)$ & $2(1,3)$ & $\begin{array}{c}38 \\
(25,3)\end{array}$ & $76(50,7)$ \\
\hline $\begin{array}{l}\text { 10. ¿Te has preocupado por el hecho de que tus } \\
\text { muslos se ensanchen cuando te sientas? }\end{array}$ & $0(0)$ & $\begin{array}{c}56 \\
(37,3)\end{array}$ & $0(0 \%)$ & $0(0 \%)$ & $45(30)$ & $49(32,7)$ \\
\hline $\begin{array}{l}\text { 11. ¿El solo hecho de comer una pequeña cantidad } \\
\text { de alimento te ha hecho sentir gorda? }\end{array}$ & $12(8)$ & $3(2)$ & $2(1,3)$ & $8(5,3)$ & $\begin{array}{c}23 \\
(15,3)\end{array}$ & $102(68)$ \\
\hline $\begin{array}{l}\text { 12. ¿Te has fijado en la figura de otros jóvenes y } \\
\text { has comparado la tuya con la de ellos } \\
\text { desfavorablemente? }\end{array}$ & $0(0)$ & $0(0)$ & $6(4)$ & $6(4)$ & $9(6)$ & $129(86)$ \\
\hline $\begin{array}{l}\text { 13. Pensar en tu figura ha interferido en tu } \\
\text { capacidad de concentración (cuando ves } \\
\text { televisión, leyendo o conversando) }\end{array}$ & $0(0)$ & $0(0)$ & $0(0)$ & $18(12)$ & $15(10)$ & $117(78)$ \\
\hline $\begin{array}{l}\text { 14. ¿Estar desnudo/a cuando te bañas ¿te ha hecho } \\
\text { sentir gordo/a? }\end{array}$ & $0(0)$ & $0(0)$ & $0(0)$ & $0(0)$ & $5(3,3)$ & $145(96,7)$ \\
\hline $\begin{array}{l}\text { 15. ¿Has evitado llevar ropa que marcasen tu } \\
\text { figura? }\end{array}$ & $0(0)$ & $10(6,7)$ & $9(6)$ & $0(0)$ & $18(12)$ & $113(75,3)$ \\
\hline $\begin{array}{l}\text { 17. ¿Comer dulces, pasteles u otros alimentos altos } \\
\text { en calorías, ¿¿te han hecho sentir gorda? }\end{array}$ & $0(0 \%)$ & $0(0)$ & $0(0)$ & $4(2,7)$ & $7(4,7)$ & $139(92,7)$ \\
\hline $\begin{array}{l}\text { 18. ¿Has evitado ir a actos sociales (por ejemplo, } \\
\text { a una fiesta) porque te has sentido mal respecto a } \\
\text { tu figura? }\end{array}$ & $0(0)$ & $0(0)$ & $0(0)$ & $11(7,3)$ & $11(7,3)$ & $128(85,3)$ \\
\hline $\begin{array}{l}\text { 19. ¿Te has sentido excesivamente gordo/a y } \\
\text { llena? }\end{array}$ & $0(0)$ & $0(0)$ & $0(0)$ & $16(10,7)$ & $15(10)$ & $119(79,3)$ \\
\hline 20. ¿'Te has sentido acomplejado/a por tu cuerpo? & $0(0)$ & $0(0)$ & $0(0)$ & $0(0)$ & $12(8)$ & $138(92)$ \\
\hline $\begin{array}{l}\text { 25. ¿Has pensado que no es justo que otras mujeres } \\
\text { sean más delgadas/os que tú? }\end{array}$ & $0(0)$ & $0(0)$ & $0(0)$ & $0(0)$ & $5(3,3)$ & $145(96,7)$ \\
\hline 26. ¿¿Has vomitado para sentirte más delgada? & $\begin{array}{c}121 \\
(80,7)\end{array}$ & $\begin{array}{c}29 \\
(19,3)\end{array}$ & $0(0)$ & $0(0)$ & $0(0)$ & $0(0)$ \\
\hline $\begin{array}{l}\text { 27. Estando en compañía de otras personas, ¿'Te ha } \\
\text { preocupado ocupar demasiado espacio (Ej. En un } \\
\text { autobús)? }\end{array}$ & $0(0)$ & $0(0)$ & $0(0)$ & $12(8)$ & $12(8)$ & $126(84)$ \\
\hline $\begin{array}{l}\text { 29. ¿Verte reflejada en un espejo ¿te hace sentir } \\
\text { mal respecto a tu figura? }\end{array}$ & $0(0)$ & $0(0)$ & $0(0)$ & $3(2)$ & $8(5,3)$ & $139(92,7)$ \\
\hline $\begin{array}{l}\text { 31. ¿ ¿Has evitado situaciones en las cuales la gente } \\
\text { pudiera ver tu cuerpo } \\
\text { (albercas, baños, vestidores)? }\end{array}$ & $0(0)$ & $0(0)$ & $0(0)$ & $6(4)$ & $12(8)$ & $132(88)$ \\
\hline $\begin{array}{l}\text { 32. ¿¿Has tomado laxantes para sentirte más } \\
\text { delgada? }\end{array}$ & $\begin{array}{c}139 \\
(92,7)\end{array}$ & $11(7,3)$ & $0(0)$ & $0(0)$ & $0(0)$ & $0(0)$ \\
\hline
\end{tabular}

Fuente: Elaboración propia 


\section{Preocupación por el peso de las universitarias}

En la Tabla 4, con respecto a la preocupación por el peso, la mayoría de las universitarias afirmaron que siempre han pensado en hacer dieta debido a la preocupación por su figura (98\%), tenido miedo en convertirse en gordas (100\%), preocupado el que su piel no sea suficientemente firme (85,3\%), sentirse gordas debido a la llenura por la comida (68,7\%), reflexionado sobre su figura al estar con gente delgada (90\%), imaginado cortándose partes de grasa de su cuerpo (72,7\%), iniciar dieta por estar preocupadas por su figura (100\%), han pensado que la figura que tienen actualmente se debe a la falta de autocontrol en la alimentación (98\%), preocupación por las celulitis $(88,7 \%)$, han pellizcado partes de su cuerpo para ver el nivel de grasa (82\%), fijarse en su figura cuando están en compañía de otros (96,7\%), y han pensado en hacer ejercicio debido a la preocupación por su figura (100\%).

Tabla 4. Criterios de preocupación por el peso

\begin{tabular}{|c|c|c|c|c|c|c|}
\hline Ítem & Nunca & Es raro & A veces & $\begin{array}{c}\text { A } \\
\text { menudo }\end{array}$ & $\begin{array}{l}\text { Muy a } \\
\text { menudo }\end{array}$ & Siempre \\
\hline & n (\%) & n (\%) & n (\%) & n (\%) & n (\%) & n (\%) \\
\hline $\begin{array}{l}\text { 2. ¿Has estado preocupada/o por tu figura } \\
\text { que has pensado que debías } \\
\text { ponerte a dieta? }\end{array}$ & $0(0)$ & $0(0)$ & $0(0)$ & $0(0)$ & $3(2)$ & $147(98)$ \\
\hline $\begin{array}{l}\text { 4. ¿ंHas tenido miedo a convertirte en } \\
\text { gorda (o más gorda)? }\end{array}$ & $0(0)$ & $0(0)$ & $0(0)$ & $0(0)$ & $0(0)$ & $150(100)$ \\
\hline $\begin{array}{l}\text { 5. ¿ } \text { Te ha preocupado el que tu piel no sea } \\
\text { suficientemente firme? }\end{array}$ & $0(0)$ & $0(0)$ & $8(5,3)$ & $10(6,7)$ & $4(2,7)$ & $128(85,3)$ \\
\hline $\begin{array}{l}\text { 6. ¿Sentirte lleno (después de la comida) te } \\
\text { ha hecho sentir gorda? }\end{array}$ & $0(0)$ & $23(15,3)$ & $10(6,7)$ & $5(3,3)$ & $9(6,0)$ & $103(68,7)$ \\
\hline $\begin{array}{l}\text { 9. ¿Estar con gente delgada te ha hecho } \\
\text { reflexionar sobre tu figura? }\end{array}$ & $0(0)$ & $0(0)$ & $0(0)$ & $0(0)$ & $15(10)$ & $135(90)$ \\
\hline $\begin{array}{l}\text { 16. ¿Te has imaginado cortando partes } \\
\text { grasas de tu cuerpo? }\end{array}$ & $0(0)$ & $0(0)$ & $0(0)$ & $0(0)$ & $41(27,3)$ & $109(72,7)$ \\
\hline $\begin{array}{l}\text { 21. ¿La preocupación por tu figura, ¿te ha } \\
\text { inducido a ponerte a dieta? }\end{array}$ & $0(0)$ & $0(0)$ & $0(0)$ & $0(0)$ & $0(0)$ & 150 (100) \\
\hline $\begin{array}{l}\text { 22. ¿Te has sentido más a gusto con tu } \\
\text { figura cuando tu estomago estaba } \\
\text { vació (por ejemplo, por la mañana)? }\end{array}$ & $0(0)$ & $0(0)$ & $0(0)$ & $76(50,7)$ & $24(16)$ & $50(33,3)$ \\
\hline $\begin{array}{l}\text { 23. ¿Has pensado que tienes la figura que } \\
\text { tienes a causa de tu falta de autocontrol? } \\
\text { (que no puedes controlar el comer menos) }\end{array}$ & $0(0)$ & $0(0)$ & $0(0)$ & $0(0)$ & $3(2)$ & 147 (98) \\
\hline $\begin{array}{l}\text { 24. ¿Te ha preocupado que la gente vea } \\
\text { "llantitas/lorzas" en tu cintura? }\end{array}$ & $76(50,7)$ & $35(23,3)$ & $18(12)$ & $0(0)$ & $9(6)$ & $12(8)$ \\
\hline $\begin{array}{l}\text { 28. ¿ } \text { Te ha preocupado que tu piel tenga } \\
\text { celulitis? }\end{array}$ & $0(0)$ & $0(0)$ & $0(0)$ & & $17(11,3)$ & $133(88,7)$ \\
\hline $\begin{array}{l}\text { 30. ¿Has pellizcado zonas de tu cuerpo } \\
\text { para ver cuanta grasa hay? }\end{array}$ & $0(0)$ & $0(0)$ & $0(0)$ & $10(6,7)$ & $17(11,3)$ & $123(82)$ \\
\hline $\begin{array}{l}\text { 33. ¿Te has fijado más en tu figura cuando } \\
\text { estas en compañía de otra gente? }\end{array}$ & $0(0)$ & $0(0)$ & $0(0)$ & $0(0)$ & $5(3,3)$ & $145(96,7)$ \\
\hline $\begin{array}{l}\text { 34. ¿La preocupación por tu figura te hace } \\
\text { pensar que deberías hacer } \\
\text { ejercicio? }\end{array}$ & $0(0)$ & $0(0)$ & $0(0)$ & $0(0)$ & $0(0)$ & $150(100)$ \\
\hline
\end{tabular}

Fuente: Elaboración propia 
Por su parte, con respecto a si se han preocupado de que la gente vea «llantitas» o lorzas en su cintura, el 50,7\% de las universitarias aseveró nunca haberse preocupado; además, en cuanto a si se han sentido más a gusto con su figura cuando su estómago estaba vacío, las universitarias respondieron mayormente muy a menudo (50,7\%).

\section{DISCUSIÓN}

De acuerdo con los resultados obtenidos con respecto a la percepción de la imagen corporal a nivel general, se identificó que el 64,7\% de las mujeres encuestadas se preocupa de manera extrema por su imagen corporal, resultado que se asocia con el obtenido en el estudio de LaraCervantes y Nieto-Mendoza (2019), donde prevaleció la «preocupación extrema por la imagen corporal» con un 92\% en mujeres de un municipio del departamento del Atlántico, Colombia. Esto indica, según Guimón (1999), una representación negativa de la imagen corporal, que puede estar asociada a experiencias dadas a partir de la relación de las universitarias con su contexto.

Este resultado, además, concuerda con diversas investigaciones ajenas al contexto abordado - México, Argentina y Chile—, donde el índice de masa corporal de las mujeres no era coherente con su realidad corporal, asociando su condición a la obesidad y el sobrepeso, lo cual denota una sobrestimación y distorsión de la imagen corporal (Soto-Montero, Guevara y Valdez, 2017; Borelli et al., 2016; Blanco et al., 2017). Por lo anterior, es preciso hacer énfasis en lo que asevera Rosen (1995), al referirse a la imagen corporal como la percepción del individuo sobre su propio cuerpo, estando el juicio que hará sobre este anclado a aspectos de carácter «perceptivos», «cognitivos afectivos» y «conductuales» (Cash et al., 1991; Thompson, 1990).

Con relación a la insatisfacción corporal, llaman la atención aspectos relacionados con el temor a la desnudez por sentirse obesas, el recurrir a comparativos desfavorables para sí con otras personas con respecto a su figura, recurrir al aislamiento por miedo al qué dirán, acomplejarse por su cuerpo, evitar situaciones en que la gente pudiese ver sus cuerpos y sentirse mal por su figura al extremo de angustiarse por ello. Estos resultados concuerdan con lo manifestado por Raich (2004), quien considera que la insatisfacción corporal — que es más frecuente en mujeres - conlleva a la autopercepción negativa, a los complejos, autorechazo, sentirse feas o deformes y recurrir a prácticas que pueden atentar contra su salud. 
No obstante, los resultados de esta investigación reflejaron una tendencia por parte de la mayoría de las universitarias a nunca haber recurrido a prácticas para sentirse delgadas, como el consumo de laxantes o prácticas como vomitar después de las comidas. Esto discrepa de lo obtenido en la investigación realizada por Torres-Rojas, Rey y Quinchucua (2017), donde las mujeres entrevistadas en la ciudad de Villavicencio (Colombia) y que se habían realizado cirugías estéticas reconocen haber recurrido a procedimientos como liposucción y lipoescultura, debido a sentirse inconformes por su imagen corporal, influenciadas por la sociedad y las nuevas tendencias del mundo globalizado.

Es de destacar también entre los resultados la manera en que incide la percepción de la imagen corporal de las universitarias en la forma de vestir, siendo entonces el complejo determinante en la selección de atuendos, escogiendo prendas fuera de su gusto por temor al qué dirán, lo cual se asocia con lo apreciado en la investigación realizada por Guayara-Cuellar (2018), donde las mujeres con antecedentes de obesidad entrevistadas afirman evitar utilizar ropa ajustada, debido a que pueden ser mayormente visibles partes de su cuerpo que les disgustan, lo cual ha conllevado además a relacionarse con pocas personas, al aislamiento y que ha desencadenado conductas que denotan depresión y ansiedad.

Con relación a la preocupación por el peso, es posible apreciar que las mujeres temen padecer de obesidad, reflexionan sobre su figura al interactuar con gente delgada y se han fijado en su figura al estar en compañía de otros; esto se asocia con lo que encontraron Lara-Cervantes y Nieto-Mendoza (2019), donde la mayoría de mujeres indagadas reconoce tener miedo a padecer obesidad, reflexionando además sobre su figura al observar a otras personas que son delgadas ya sea en la televisión, en su contexto o con personas con las que interactúan, recurriendo a comparativos con respecto a su peso corporal que resultan casi siempre negativos para sí mismas. Cabe precisar que, diversas investigaciones han dado cuenta de la disociación entre el peso corporal real de las mujeres y su percepción de la imagen corporal, lo que se debe a ideas infundadas por la publicidad, la sociedad y los medios de comunicación (GonzálezGonzález de Mesa et al., 2016; Guzmán-Saldaña et al., 2017; Trejo-Ortiz et al., 2016; ZueckEnríquez et al., 2016; Soto-Montero, Guevara-Valtier y Valdez-Montero, 2017; Sim-Sim et al., 2018).

Hay que tener presente también aspectos palmarios en la investigación con relación a la tendencia de las mujeres a la práctica de actividad física y dietas al sentirse preocupadas por su figura y reconociendo que la falta de autocontrol a la hora de ingerir alimentos ha incidido negativamente en su figura; esto puede ser aprovechado para la implementación de estrategias 
desde el entorno universitario que induzcan a la práctica regular de actividad física y crear campañas de sensibilización con respecto a la alimentación sana y pautas para el cuidado del cuerpo, puesto que, a pesar de que la American Psychiatric Association (1994) afirme que el exceso de preocupación por la imagen corporal puede traer consigo desórdenes alimentarios, también reconoce que esta puede ser una ventaja para la promoción de estilos de vida saludables por medio de la concienciación sobre las implicaciones que trae consigo recurrir a prácticas que pueden poner en riesgo su vida.

Por otra parte, precisando en la limitaciones de la investigación, debido a las problemáticas que afrontó la Universidad del Atlántico, Barranquilla, Colombia en el semestre académico 2019-2 que impidieron el flujo académico normal, no fue posible considerar aplicar el cuestionario a una mayor cantidad de mujeres del mismo programa, lo cual hubiese representado resultados de mayor impacto; asimismo, no fue posible realizar un contraste entre la percepción de la imagen corporal y el índice de masa corporal (IMC) de las universitarias, siendo determinante para identificar si la imagen que tienen de su cuerpo las estudiantes concuerda con su peso corporal real.

Finalmente, se proponen como líneas de investigación futuras realizar un estudio correlacional entre la imagen corporal entre hombres y mujeres que pretenda dar a conocer qué genero presenta mayor preocupación por cómo se ven y sienten con su cuerpo; además, se pretende establecer relación entre las variables «imagen corporal» e «índice de masa corporal» con el fin de identificar la sobreestimación de las universitarias de su peso corporal.

\section{CONCLUSIONES}

La Universidad del Atlántico debe comprometerse a garantizar un contexto universitario saludable basado en el fomento de la actividad física y la conformación de planes de valoración de índice de masa corporal en la población femenina del entorno estudiado, con el fin de brindar pautas eficientes y coherentes con el estado nutricional de los individuos. Para ello, debe servirse de la cooperación de nutricionistas y entrenadores deportivos coordinados por la oficina de Bienestar Universitario que realicen proyectos que sean interesantes para la población universitaria, como actividades de carácter recreativo y lúdico que estén al servicio del desarrollo integral de las mujeres y sirvan para la modificación de conductas insanas que repercuten en su imagen corporal. 


\section{BIBLIOGRAFÍA}

American Psychiatric Association. Diagnostic and statistical manual of mental disorders, dsm-iv. APA, 1994. [Consulta: 18 de febrero de 2020]. Disponible en: www.researchgate.net/publication/230002115_diagnostic_and_statistical_manual_o f_mental_disorders_dsm-iv-tr

AmezcuA, Valeria. Cifras de anorexia y bulimia en América Latina. 28 de julio de 2014. [Consulta: 18 de febrero de 2020]. Disponible en:

http://kualifamily.com/obesidad/consejos-obesidad/cifras-de-anorexia-y-bulimiaen-america-latina/

BLANCO, Humberto, et al. Actividad física, imagen corporal y autoconcepto personal en jóvenes. En: Revista de Psicología del Deporte. 2017, vol. 26, núm. 2, pp. 25-33.

BORDA-PÉREZ, Mariela, et al. Percepción de la imagen corporal y su relación con el estado nutricional y emocional en escolares de 10 a 13 años de tres escuelas en Barranquilla (Colombia). En: Salud Uninorte. 2016, vol. 32, pp. 472-482.

Borelli, María, et al. Estado Nutricional y Percepción de la Imagen Corporal de Embarazadas asistidas en Centros de Salud de Salta Capital, Argentina. En: Revista Española de nutrición Humana y Dietética. 2016, vol. 20, núm. 3, pp. 174-179.

CAsH, Thomas, et al. Body images: development, deviance and changes. En: Plastic and Reconstructive Surgery. 1991, vol. 88, núm. 2, p. 367.

CASTRILLÓn, Diego, et al. Validación del Body Shape Questionnaire (cuestionario de la figura corporal) BSQ para la población colombiana. En: Acta colombiana de psicología. 2007, vol. 10, núm. 1, pp. 15-23

COOPER, Peter, et al. The development and validation of the Body Shape Questionnaire. En: International Journal of Eating Disorders. 1987, vol. 6, pp. 485-94.

Duno, Maryelin; AcostA, Edgar. Percepción de la imagen corporal en adolescentes universitarios. En: Revista Chilena de Nutrición. 2019, vol. 46, núm, 5, pp. 543-553. ESPINOZA-LARA, Olga. Hábitos alimentarios, percepción de la imagen corporal y estado nutricional en mujeres adolescentes de 14 a 16 años del Colegio María Magdalena Ortega de Nariño Bogotá D.C. [Tesis de pregrado]. Bogotá: Pontificia Universidad Javeriana, 2014. [Consulta: 18 de febrero de 2020]. Disponible en: https://repository.javeriana.edu.co/bitstream/handle/10554/16022/EspinosaLaraOlg aPatricia2014.pdf?sequence $=1$ \&isAllowed $=\mathrm{y}$ 
Florez-Alvarez, Sergio. Percepción de la imagen corporal de adolescentes escolares de 12 a 19 años en colegios públicos. [Tesis de pregrado]. Bogotá: Universidad de Santander (UDES), 2017. [Consulta: 18 de febrero de 2020]. Disponible en: https://repositorio.udes.edu.co/bitstream/001/96/1/PercepciBucaramanga.pdf

GonZÁLEZ-GonZÁLEz DE MESA, Carmen, et al. Relación entre variables demográficas, la práctica de ejercicio físico y la percepción de la imagen corporal en estudiantes del grado de magisterio. En: RETOS. Nuevas Tendencias en Educación Física, Deporte y Recreación. 2016, vol. 29, pp. 90-94.

GuAYARA-Cuellar, Ángela. Autoconcepto en mujeres en situación de obesidad. [Tesis de pregrado]. Universidad Surcolombiana, 2018. [Consulta: 18 de febrero de 2020]. Disponible en: http://repositorio.usco.edu.co/handle/123456789/573

Guimón, José. Los lugares del cuerpo. Barcelona: Paidós, 1999.

GUZMÁN-SALDAÑA, Rebeca, et al. Imagen corporal e índice de masa corporal en mujeres indígenas del estado. En: Revista Mexicana de trastornos alimentarios. 2017, vol. 8, núm. 1, pp. 56-62.

Lara-Cervantes, Bruno; Nieto-MendozA, Isaac. Percepción de la imagen corporal en mujeres de 25 años del municipio de ponedera (Atlántico). En: Biociencias. 2019. vol. 14, núm. 4, pp. 113-127.

LóPez, Carolina; Treasure, Janet. Trastornos de la conducta alimentaria en adolescentes: descripción y manejo. En: Revista Médica Clínica CONDES. 2011, vol. 22, núm. 1, pp. 85-97.

Ministerio de SAlud y Protección Social. Resolución 8430. Por la cual se establecen las normas científicas, técnicas y administrativas para la investigación en salud: 4 de octubre de 1993. [Consulta: 14 de noviembre de 2019]. Disponible en: https://www.minsalud.gov.co/sites/rid/lists/bibliotecadigital/ride/de/dij/resolucion8430-de-1993.pdf

NiETO-Ortiz, Dolores; Nieto-MendozA, Isaac. Estilo de vida de estudiantes de la Universidad del Atlántico: un estudio descriptivo-transversal. En: Ciencia $e$ Innovación en Salud. 2020, pp. 1-16.

NiETO-OrTIZ, Dolores; TORRENEgrA-ARIZA, Silvia. Relación entre el índice de masa corporal y actividad física en universitarios de la ciudad de Barranquilla en el semestre 2018-2, Universidad del Atlántico. En: Biociencias. 2019, vol. 14, núm.1, pp. 151-164. 
RAICH, Rosa. Una perspectiva desde la psicología de la salud de la imagen corporal. En: Avances de psicología latinoamericana. 2004, vol. 22, núm. 1, pp. 15-27.

RinCÓN-BARRETO, Dubis, et al. Percepción de la Imagen corporal en universitarios de la Ciudad de Medellín. En: Psicoespacios. 2019, vol. 13, núm. 23, pp. 62-70.

Rosen, James. The nature of body dysmorphic disorder and treatment whit cognitive behavior therapy. En: Cognitive and Behavioral Practice. 1995, vol. 2, núm. 1, pp. 143-166.

SAlAZAR-Duque, Beatriz. Percepción de la imagen corporal en Embera Chamí: dimensiones corporales. En: Revista Jangwa Pana. 2015, vol. 14, núm. 1, pp. 34-45.

SANTOS, Gabriel, et al. Percepción de la imagen corporal en relación a la composición corporal en estudiantes de la Universidad de Vigo. Alicante: Universidad de Alicante, 2017. [Consulta: 18 de febrero de 2020]. Disponible en:

\section{http://rua.ua.es/dspace/handle/10045/67305}

SiM-SIM, Margarita, et al. Percepción de la Imagen Corporal en embarazadas en el tercer trimestre. En: RIASE. 2018, vol. 4, núm. 1, pp. 1310-1321.

Soto-Montero, Fabiola; Guevara-Vatier, Milton; VALdeZ-Montero, Carolina. Composición y percepción de la imagen corporal en mujeres adultas. En: Ra Ximhai. 2017, vol. 13, núm. 2, pp. 97-103.

ThOMPSON, Joel. Body image disturbances: Assessment and treatment. New York: Pergamon Press, 1990.

TORREs-RojAs, Daniela, et al. Autoconcepto en mujeres jóvenes consumidoras de cirugías plásticas estéticas de la ciudad de Villavicencio. [Tesis de pregrado]. Universidad Cooperativa de Colombia, 2017. [Consulta: 18 de febrero de 2020]. Disponible en:

https://repository.ucc.edu.co/bitstream/20.500.12494/4443/2/2017_autoconcepto_m ujeres_consumidoras.pdf

Trejo-OrTiz, Perla, et al. Hábitos de actividad física y cánones de imagen corporal en estudiantes universitarios. En: Revista Cubana de Medicina General Integral. 2016, vol. 32, núm. 1, pp. 72-82.

ZuECK-ENRÍQUEZ, María, et al. Imagen corporal en universitarios mexicanos: diferencias entre hombres y mujeres. En: Retos: nuevas tendencias en educación física, deporte y recreación. 2016, núm. 30, pp. 171-176. 